\title{
DIFFERENT ASYMPTOTIC SPREADING SPEEDS INDUCED BY ADVECTION IN A DIFFUSION PROBLEM WITH FREE BOUNDARIES
}

\author{
HONG GU, ZHIGUI LIN, AND BENDONG LOU
}

(Communicated by Yingfei Yi)

\begin{abstract}
In this paper, we consider a Fisher-KPP equation with an advection term and two free boundaries, which models the behavior of an invasive species in one dimension space. When spreading happens (that is, the solution converges to a positive constant), we use phase plane analysis and upper/lower solutions to prove that the rightward and leftward asymptotic spreading speeds exist and both are positive constants. Moreover, one of them is bigger and the other is smaller than the spreading speed in the corresponding problem without advection term.
\end{abstract}

\section{INTRODUCTION}

In 2010, Du and Lin [6] studied the following Fisher-KPP problem with free boundaries:

$$
\begin{cases}u_{t}-d u_{x x}=u(1-u), & g(t)<x<h(t), t>0 \\ u(t, g(t))=0, \quad g^{\prime}(t)=-\mu u_{x}(t, g(t)), & t>0 \\ u(t, h(t))=0, \quad h^{\prime}(t)=-\mu u_{x}(t, h(t)), & t>0 \\ -g(0)=h(0)=h_{0}, \quad u(0, x)=u_{0}(x), & -h_{0} \leq x \leq h_{0}\end{cases}
$$

where $d$ and $\mu$ are positive constants, the initial function $u_{0}(x)$ satisfies

$$
u_{0} \in C^{2}\left(\left[-h_{0}, h_{0}\right]\right), \quad u_{0}\left( \pm h_{0}\right)=0 \text { and } u_{0}>0 \text { in }\left(-h_{0}, h_{0}\right),
$$

for some $h_{0}>0$. They used $\left(P_{0}\right)$ to model the spreading of a new or invasive species with population density $u(t, x)$ over a one dimensional habitat, with the free boundaries $x=g(t), h(t)$ representing the expanding fronts. They obtained a dichotomy result: that is, either spreading happens $(u(t, \cdot) \rightarrow 1$ locally uniformly in $\mathbb{R}$ and $h(t),-g(t) \rightarrow \infty$ as $t \rightarrow \infty)$ or vanishing happens $(u(t, \cdot) \rightarrow 0$ uniformly in $[g(t), h(t)]$ as $t \rightarrow \infty$ and $\left.\lim _{t \rightarrow \infty}[h(t)-g(t)]<\infty\right)$. Furthermore, when spreading happens, they obtained the existence of the asymptotic spreading speed ([6. Proposition 4.1]):

$$
c^{*}:=\lim _{t \rightarrow \infty} \frac{h(t)}{t}=\lim _{t \rightarrow \infty} \frac{-g(t)}{t}>0 .
$$

Recently, further extensions have been done; for example, Du and Guo [4,5] studied the problem in higher dimension spaces and in heterogeneous environment. Du

Received by the editors January 13, 2013 and, in revised form, March 27, 2013.

2010 Mathematics Subject Classification. Primary 35B40, 35K57, 34C37, 92B05.

This paper was partly supported by the NSFC (11271285, 11071209). 
and Lou [7] studied the problem with general nonlinear $f$, including general monostable, bistable and combustion types of $f$. Among others, they all proved that the asymptotic spreading speed when spreading happens is the same positive constant in any direction.

However, some species prefers to move towards one direction because of rich resources, appropriate climate, etc. Some diseases spread along the wind direction. In 2009, Maidana and Yang 9] studied the propagation of West Nile Virus from New York City to California. It was observed that West Nile Virus appeared for the first time in New York City in the summer of 1999. In the second year the wave front traveled $187 \mathrm{~km}$ to the north and $1100 \mathrm{~km}$ to the south. Therefore, they took account of the advection movement and showed that bird advection becomes an important factor in lowering mosquito biting rates. Recently, Averill [1] considered the effect of intermediate advection on the dynamics of a two-species competition system, and provides a concrete range of advection strength for the coexistence of two competing species. Moreover, three different kinds of transitions from small advection to large advection were illustrated theoretically and numerically.

What is the difference between the asymptotic spreading speed of the left frontier and that of the right frontier when an invasive species is spreading? To address the question, in this paper we study the following problem with an advection term:

$$
\begin{cases}u_{t}-d u_{x x}+\beta u_{x}=u(1-u), & g(t)<x<h(t), t>0, \\ u(t, g(t))=0, \quad g^{\prime}(t)=-\mu u_{x}(t, g(t)), & t>0, \\ u(t, h(t))=0, \quad h^{\prime}(t)=-\mu u_{x}(t, h(t)), & t>0, \\ -g(0)=h(0)=h_{0}, \quad u(0, x)=u_{0}(x), & -h_{0} \leq x \leq h_{0},\end{cases}
$$

where $d, \mu, h_{0}, u_{0}$ are as above and $\beta>0$ is a constant.

By a similar argument as in [4, 6, 7, we have the following basic results.

(i) Problem $\left(P_{1}\right.$ has a time global solution $(u, g, h)$ with $u \in C^{1+\alpha / 2,2+\alpha}\left(G_{T}\right)$ and $g, h \in C^{1+\alpha / 2}((0, T])$ for any $T>0$ and any $\alpha \in(0,1)$, where

$$
G_{T}=\{(t, x): g(t) \leq x \leq h(t), 0<t \leq T\} ;
$$

(ii) $0<u(t, x) \leq C_{1}$ for $g(t)<x<h(t), t>0$ and $0<-g^{\prime}(t), h^{\prime}(t)<C_{2}$ for $t>0$, where $C_{1}$ and $C_{2}$ are constants independent of $t$.

In a forthcoming paper [8], we study the asymptotic behavior of the solutions of $P_{1}$. More precisely, we give some sufficient conditions for spreading and some sufficient conditions for vanishing. It turns out that spreading happens only if

$$
0<\beta<2 \sqrt{d} \text {. }
$$

This paper is devoted to the difference between the leftward and rightward asymptotic spreading speeds induced by the advection term $\beta u_{x}$. This is an interesting problem from an ecological point of view.

Theorem 1.1. Assume $0<\beta<2 \sqrt{d}$. Let $(u, g, h)$ be a solution of $\left(P_{1}\right.$ for which spreading happens. Then the leftward and rightward asymptotic spreading speeds exist:

$$
c_{l}^{*}:=\lim _{t \rightarrow \infty} \frac{-g(t)}{t}, \quad c_{r}^{*}:=\lim _{t \rightarrow \infty} \frac{h(t)}{t} .
$$

Moreover, $0<c_{l}^{*}<c^{*}<c_{r}^{*}$, where $c^{*}$ is the spreading speed of the solution of $P_{0}$.

$c^{*}$ is given in (1.2) which is nothing but $k_{0}$ in [6, Proposition 4.1], or $c^{*}$ in [7, Theorem 1.10]. It depends on the parameter $\mu$. Similarly $c_{l}^{*}$ and $c_{r}^{*}$ depend on $\mu$. 
Clearly, $c_{r}^{*}$ and $c_{l}^{*}$ also depend on $\beta$. On these dependences we have the following results.

Theorem 1.2. in $\mu$, and

(i) If $\beta \in(0,2 \sqrt{d})$ is fixed, then $c_{l}^{*}, c^{*}, c_{r}^{*}$ are strictly increasing

$$
\begin{array}{ll}
\lim _{\mu \rightarrow 0} c_{l}^{*}=0, & \lim _{\mu \rightarrow \infty} c_{l}^{*}=2 \sqrt{d}-\beta, \\
\lim _{\mu \rightarrow 0} c^{*}=0, & \lim _{\mu \rightarrow \infty} c^{*}=2 \sqrt{d}, \\
\lim _{\mu \rightarrow 0} c_{r}^{*}=0, & \lim _{\mu \rightarrow \infty} c_{r}^{*}=2 \sqrt{d}+\beta ;
\end{array}
$$

(ii) if $\mu$ is fixed, then $c_{r}^{*},-c_{l}^{*}$ are strictly increasing in $\beta$, and

$$
\lim _{\beta \rightarrow 0} c_{l}^{*}=\lim _{\beta \rightarrow 0} c_{r}^{*}=c^{*}, \lim _{\beta \rightarrow 2 \sqrt{d}} c_{l}^{*}=0 .
$$

\section{SEMI-WAVES AND SPREADING SPEEDS}

Throughout this section we assume that (1.3) holds and that $(u, g, h)$ is a solution of $\left(P_{1}\right)$ for which spreading happens, that is, $h(t),-g(t) \rightarrow \infty(t \rightarrow \infty)$, and $u(t, \cdot) \rightarrow 1$ locally uniformly in $\mathbb{R}$. Denote $f(u):=u(1-u)$ for convenience. We remark that the approaches below remain valid for general monostable nonlinear $f$.

To determine the spreading speed, we construct upper and lower solutions based on semi-waves.

2.1. Phase plane analysis and semi-waves. We call $q(z)$ a semi-wave with speed $c$ if $(c, q(z))$ satisfies

$$
\left\{\begin{array}{l}
q^{\prime \prime}-\frac{c-\beta}{d} q^{\prime}+\frac{f(q)}{d}=0 \quad \text { for } z \in(0, \infty) \\
q(0)=0, q(\infty)=1, q(z)>0 \text { for } z \in(0, \infty) .
\end{array}\right.
$$

The first equation in this problem is equivalent to the following system:

$$
\left\{\begin{array}{l}
q^{\prime}=p, \\
p^{\prime}=\frac{c-\beta}{d} p-\frac{f(q)}{d} .
\end{array}\right.
$$

A solution $(q(z), p(z))$ of this system traces out a trajectory in the $q, p$-plane or, as it is usually called, the phase plane (cf. 2, 3, 7, 10 ). Such a trajectory has slope

$$
\frac{\mathrm{d} p}{\mathrm{~d} q}=\frac{c-\beta}{d}-\frac{f(q)}{d p}
$$

at any point where $p \neq 0$. Here we are only interested in a trajectory of (2.2) that starts from the point $(0, \omega)$ with some $\omega \geq 0$ and ends at the point $(1,0)$ as $z \rightarrow+\infty$.

For any fixed $c \geq 0,(0,0)$ and $(1,0)$ are critical points of the system (2.2). The eigenvalues of the corresponding linearizations are

$$
\begin{aligned}
& \lambda_{0}^{ \pm}=\frac{c-\beta \pm \sqrt{(c-\beta)^{2}-4 d}}{2 d}(\text { at }(0,0)), \\
& \lambda_{1}^{ \pm}=\frac{c-\beta \pm \sqrt{(c-\beta)^{2}+4 d}}{2 d}(\text { at }(1,0)),
\end{aligned}
$$

respectively. Thus $(1,0)$ is a saddle point and $(0,0)$ is 
(i) a center or a spiral point, if $0 \leq c<\beta+2 \sqrt{d}$;

(ii) a nodal point, if $c \geq \beta+2 \sqrt{d}$.

Therefore, by the theory of ordinary differential equations (ODE) (cf. [10]), there are exactly two trajectories of (2.2) that approach $(1,0)$ from $q<1$. One of them, denoted by $T_{r}^{c}$, has slope $\lambda_{1}^{-}<0$ at $(1,0)$. Suppose that $T_{r}^{c}$ is expressed by a function $p=P_{r}^{c}(q)$. Then $p=P_{r}^{c}(q)$ satisfies (2.3) and $T_{r}^{c}$ lies in the semistrip

$$
S=\{(q, p): 0<q<1, p>0\} .
$$

$T_{r}^{c}$ is a trajectory through $(1,0)$ and $\left(0, P_{r}^{c}\left(0^{+}\right)\right)$for some $P_{r}^{c}\left(0^{+}\right) \geq 0$. The following are well-known results (cf. [2, 3, 7, 11]).

Proposition 2.1. Let $c_{r}^{0}:=2 \sqrt{d}+\beta$. Then

(i) for any $c \in\left[0, c_{r}^{0}\right), P_{r}^{c}(0)$ is positive, continuous, strictly decreasing in $c$, and $\lim _{c \nearrow c_{r}^{0}} P_{r}^{c}(0)=0$

(ii) for any $c \geq c_{r}^{0}, P_{r}^{c}\left(0^{+}\right)=0$.

In case (ii), each $T_{r}^{c}$ is a trajectory in $S$ through $(0,0)$ and $(1,0)$, and so it corresponds to a traveling wave with speed $c, c_{r}^{0}$ is nothing but the minimal speed of these traveling waves.

Denote $\zeta(c):=P_{r}^{c}(0)-\frac{c}{\mu}$ for $c \in\left[0, c_{r}^{0}\right)$. In view of Proposition 2.1. $\zeta(c)$ is continuous and strictly decreasing in $c \in\left[0, c_{r}^{0}\right)$, and it satisfies

$$
\begin{aligned}
& \zeta(0)=P_{r}^{0}(0)>P_{r}^{\beta}(0)=\sqrt{\frac{2}{d} \int_{0}^{1} f(s) d s}>0, \\
& \zeta\left(\left(c_{r}^{0}\right)^{-}\right)=-\frac{c_{r}^{0}}{\mu}<0 .
\end{aligned}
$$

Thus there exists a unique $c_{r}^{*} \in\left(0, c_{r}^{0}\right)$ such that $\zeta\left(c_{r}^{*}\right)=0$, i.e. $P_{r}^{c_{r}^{*}}(0)=c_{r}^{*} / \mu$. Summarizing the above results we have the following proposition.

Proposition 2.2. Problem (2.1) has exactly one solution $(c, q)=\left(c_{r}^{*}, q_{r}^{*}\right)$ such that

$$
\mu\left(q_{r}^{*}\right)^{\prime}(0)=c_{r}^{*} .
$$

Moreover, $c_{r}^{*} \in(0, \beta+2 \sqrt{d})$.

Later we will use this semi-wave to estimate the rightward spreading speed. Similarly, to estimate the leftward spreading speed, we will need another semi-wave traveling to the left, which is a solution of the following problem:

$$
\left\{\begin{array}{l}
q^{\prime \prime}-\frac{c+\beta}{d} q^{\prime}+\frac{f(q)}{d}=0 \quad \text { for } z \in(0, \infty) \\
q(0)=0, q(\infty)=1, q(z)>0 \text { for } z \in(0, \infty) .
\end{array}\right.
$$

Similarly as above, this problem can be studied by considering the problem

$$
\frac{\mathrm{d} p}{\mathrm{~d} q}=\frac{c+\beta}{d}-\frac{f(q)}{d p}
$$

in the $q, p$-phase plane, where $p=q^{\prime}$. Denote $P_{l}^{c}(q)$ the solution of this equation whose trajectory through $(1,0)$ and $\left(0, P_{l}^{c}\left(0^{+}\right)\right)$for some $P_{l}^{c}\left(0^{+}\right) \geq 0$. Similarly as above, we have the following results. 
Proposition 2.3. Let $c_{l}^{0}:=2 \sqrt{d}-\beta$. Then

(i) for any $c \in\left[0, c_{l}^{0}\right), P_{l}^{c}(0)$ is positive, continuous, strictly decreasing in $c$, and $\lim _{c \nearrow c_{l}^{0}} P_{l}^{c}(0)=0$;

(ii) for any $c \geq c_{l}^{0}, P_{l}^{c}\left(0^{+}\right)=0$.

Proposition 2.4. Problem (2.5) has exactly one solution $(c, q)=\left(c_{l}^{*}, q_{l}^{*}\right)$ such that

$$
\mu\left(q_{l}^{*}\right)^{\prime}(0)=c_{l}^{*} .
$$

Moreover, $c_{l}^{*} \in\left(0, c_{l}^{0}\right)$.

Next, we make suitable perturbations of $f(u)$ to derive corresponding semi-waves that can be used to construct upper and lower solutions of $\left(P_{1}\right.$.

For any small $\varepsilon>0$, set

$$
\begin{aligned}
& \widetilde{f}_{\varepsilon}(u):=f(u)-\frac{\varepsilon}{1-\varepsilon} u^{2} \equiv u\left(1-\frac{1}{1-\varepsilon} u\right), \\
& \widehat{f}_{\varepsilon}(u):=f(u)+\frac{\varepsilon}{1+\varepsilon} u^{2} \equiv u\left(1-\frac{1}{1+\varepsilon} u\right) .
\end{aligned}
$$

Note that $\tilde{f}_{\varepsilon}(u)$ is strictly decreasing in $\varepsilon$ and it has exactly two zeros, 0 and $1-\varepsilon$. $\widehat{f}_{\varepsilon}(u)$ is strictly increasing in $\varepsilon$ and it has exactly two zeros, 0 and $1+\varepsilon$. Similarly as above, we know that problem (2.1) with $f$ replaced by $\widetilde{f}_{\varepsilon}$ (resp. $\widehat{f}_{\varepsilon}$ ) has exactly one solution $\left(\widetilde{c}_{r}^{*}, \widetilde{q}_{r}^{*}\right)$ with $\mu\left(\widetilde{q}_{r}^{*}\right)^{\prime}(0)=\widetilde{c}_{r}^{*}$ and $\widetilde{c}_{r}^{*} \in\left(0, c_{r}^{0}\right)$ (resp. a solution $\left(\widehat{c}_{r}^{*}, \widehat{q}_{r}^{*}\right)$ with $\mu\left(\widehat{q}_{r}^{*}\right)^{\prime}(0)=\widehat{c}_{r}^{*}$ and $\left.\widehat{c}_{r}^{*} \in\left(0, c_{r}^{0}\right)\right)$, where $c_{r}^{0}=2 \sqrt{d}+\beta$. Similarly, problem (2.5) with $f$ replaced by $\widetilde{f}_{\varepsilon}$ (resp. $\left.\widehat{f}_{\varepsilon}\right)$ has exactly one solution $\left(\widetilde{c}_{l}^{*}, \widetilde{q}_{l}^{*}\right)$ with $\mu\left(\widetilde{q}_{l}^{*}\right)^{\prime}(0)=\widetilde{c}_{l}^{*}$ and $\widetilde{c}_{l}^{*} \in\left(0, c_{l}^{0}\right)$ (resp. a solution $\left(\widehat{c}_{l}^{*}, \widehat{q}_{l}^{*}\right)$ with $\mu\left(\widehat{q}_{l}^{*}\right)^{\prime}(0)=\widehat{c}_{l}^{*}$ and $\left.\widehat{c}_{l}^{*} \in\left(0, c_{l}^{0}\right)\right)$, where $c_{l}^{0}=2 \sqrt{d}-\beta$.

Proposition 2.5. The following conclusions hold.

$$
\widetilde{c}_{r}^{*}<c_{r}^{*}<\widehat{c}_{r}^{*}, \quad \lim _{\varepsilon \rightarrow 0} \widetilde{c}_{r}^{*}=\lim _{\varepsilon \rightarrow 0} \widehat{c}_{r}^{*}=c_{r}^{*},
$$

and

$$
\widetilde{c}_{l}^{*}<c_{l}^{*}<\widehat{c}_{l}^{*}, \quad \lim _{\varepsilon \rightarrow 0} \widetilde{c}_{l}^{*}=\lim _{\varepsilon \rightarrow 0} \widehat{c}_{l}^{*}=c_{l}^{*} .
$$

Proof. We first prove $\widetilde{c}_{r}^{*}<c_{r}^{*}$. For any $c \in\left[0, c_{r}^{0}\right)$, consider the problem (2.3) with $f$ replaced by $\widetilde{f}_{\varepsilon}$, denote the solution with trajectory through the critical point $(0,1-\varepsilon)$ in the phase plane by $\widetilde{P}_{r, \varepsilon}^{c}(q)$. Similarly to Proposition 2.1 (i) we have $\widetilde{P}_{r, \varepsilon}^{c}(0)>0$ for all $c \in\left[0, c_{r}^{0}\right)$. Moreover, $\widetilde{P}_{r, \varepsilon}^{c}(q)<P_{r}^{c}(q)(q \in(0,1-\varepsilon])$ by $\tilde{f}_{\varepsilon}(q) \leq f(q)(0<q \leq 1-\varepsilon)$. We now prove

$$
0<\widetilde{P}_{r, \varepsilon}^{c}(0)<P_{r}^{c}(0) \text { for } c \in\left[0, c_{r}^{0}\right) .
$$

Otherwise, $\widetilde{P}_{r, \varepsilon}^{c}(0)=P_{r}^{c}(0)$, and so the function $\eta(q):=P_{r}^{c}(q)-\widetilde{P}_{r, \varepsilon}^{c}(q)$ satisfies

$$
\eta^{\prime}<a(q) \eta \quad(0<q<1-\varepsilon), \quad \eta(0)=0,
$$

where $a(q):=\tilde{f}_{\varepsilon}(q)\left[d P_{r}^{c}(q) \widetilde{P}_{r, \varepsilon}^{c}(q)\right]^{-1}$. This implies that $\eta(q)<0(0<q<1-\varepsilon)$, a contradiction.

Denote $\tilde{\zeta}(c):=\widetilde{P}_{r, \varepsilon}^{c}(0)-\frac{c}{\mu}$. Then (2.8) implies that

$$
\tilde{\zeta}(c)<\zeta(c) \text { for } c \in\left[0, c_{r}^{0}\right) \text {. }
$$


Similarly as above, both $\tilde{\zeta}(c)$ and $\zeta(c)$ are continuous and strictly decreasing functions in $\left[0, c_{r}^{0}\right)$, and

$$
\tilde{\zeta}\left(\left(c_{r}^{0}\right)^{-}\right)=\zeta\left(\left(c_{r}^{0}\right)^{-}\right)=-\frac{c_{r}^{0}}{\mu} .
$$

Therefore $\widetilde{c}_{r}^{*}<c_{r}^{*}$ by their definitions: $\tilde{\zeta}\left(\widetilde{c}_{r}^{*}\right)=\zeta\left(c_{r}^{*}\right)=0$.

Next we prove $\lim _{\varepsilon \rightarrow 0} \widetilde{c}_{r}^{*}=c_{r}^{*}$. It is sufficient to show that, for any $c \in\left[0, c_{r}^{0}\right)$,

$$
\widetilde{P}_{r, \varepsilon}^{c}(0) \rightarrow P_{r}^{c}(0) \text { as } \varepsilon \rightarrow 0 \text {. }
$$

By the monotonicity of $\tilde{f}_{\varepsilon}$, it is easily seen that $\widetilde{P}_{r, \varepsilon}^{c}(q)$ is monotonically decreasing in $\varepsilon$, and it is bounded from above by $P_{r}^{c}(q)$. Therefore, as $\varepsilon \rightarrow 0, \widetilde{P}_{r, \varepsilon}^{c}(q)$ converges to some function $R(q)$ in $C^{1}([0,1-\delta])$ for any $0<\delta<1$. Clearly, $p=R(q)$ corresponds to a trajectory of (2.2) that approaches $(1,0)$ in the phase plane with a non-positive slope at $(1,0)$. Consequently, $R(q) \equiv P_{r}^{c}(q)$, and so (2.9) is proved.

Other conclusions can be proved in a similar way as above.

\subsection{Asymptotic spreading speed.}

Proof of Theorem 1.1. We first estimate the rightward asymptotic spreading speed. For any small $\varepsilon>0$ we define

$$
\widetilde{w}(t, x):=\widetilde{q}_{r}^{*}\left(\widetilde{c}_{r}^{*} t-x\right), \quad x \in\left[0, \widetilde{c}_{r}^{*} t\right],
$$

Since $\left(\widetilde{c_{r}^{*}}, \widetilde{q}_{r}^{*}(z)\right)$ satisfies

$$
\left\{\begin{array}{l}
q^{\prime \prime}-\frac{c-\beta}{d} q^{\prime}+\frac{\widetilde{f}_{\varepsilon}(q)}{d}=0 \quad \text { for } z \in(0, \infty) \\
q(0)=0, q(\infty)=1-\varepsilon, q^{\prime}(0)=\frac{c}{\mu} \text { and } q^{\prime}(z)>0(z>0)
\end{array}\right.
$$

we have

$$
\widetilde{w}(t, x) \leq 1-\varepsilon, \quad \widetilde{w}_{t}-\widetilde{w}_{x x}+\beta \widetilde{w}_{x}=\widetilde{f}_{\varepsilon}(\widetilde{w}) \quad \text { for } x \in\left[0, \widetilde{c}_{r}^{*} t\right], t>0,
$$

and

$$
\widetilde{w}\left(t, \widetilde{c}_{r}^{*} t\right)=0, \quad \widetilde{c}_{r}^{*}=-\mu \widetilde{w}_{x}\left(t, \widetilde{c}_{r}^{*} t\right) \quad \text { for } t \geq 0 .
$$

Since we are considering the spreading case, we have $\lim _{t \rightarrow \infty} u(t, \cdot)=1$ locally uniformly in $\mathbb{R}$. In particular,

$$
u(t, 0)>1-\varepsilon \text { for } t>T
$$

for some $T>0$. Thus $\left(\widetilde{w}(t, x), \widetilde{c}_{r}^{*} t\right)$ is a lower solution of $P_{1}$ on $\{(t, x) \mid x \in$ $\left.\left[0, \widetilde{c}_{r}^{*} t\right], t>0\right\}$ by comparison principle (cf. [6, 7]), and

$$
\widetilde{c}_{r}^{*} t \leq h(t+T), \quad \widetilde{w}(t, x) \leq u(t+T, x) \quad \text { in }\left\{(t, x) \mid x \in\left[0, \widetilde{c}_{r}^{*} t\right], t>0\right\} .
$$

This implies that

$$
\liminf _{t \rightarrow \infty} \frac{h(t)}{t} \geq \widetilde{c}_{r}^{*}
$$

Next we estimate the upper bound of the rightward spreading speed. Consider the problem

$$
\eta^{\prime}(t)=f(\eta) \quad(t>0), \quad \eta(0)=\left\|u_{0}\right\|_{\infty}+1 .
$$

A simple comparison shows that

$$
u(t, x) \leq \eta(t):=\left(1-\frac{\left\|u_{0}\right\|_{\infty}}{\left\|u_{0}\right\|_{\infty}+1} e^{-t}\right)^{-1} \quad \text { for } x \in[g(t), h(t)], t>0 .
$$


Hence for any small $\varepsilon>0$, there exists $\widehat{T}>0$ such that

$$
u(t, x) \leq 1+\frac{\varepsilon}{2} \quad \text { for } x \in[0, h(t)], t \geq \widehat{T} .
$$

Recall that $\left(\widehat{c}_{r}^{*}, \widehat{q}_{r}^{*}(z)\right)$ is a solution of problem (2.1) with $f$ replaced by $\widehat{f}_{\varepsilon}$ and $\widehat{q}_{r}^{*}(\infty)=1+\varepsilon$. Hence there exists $\widehat{x}>h(\widehat{T})$ large such that

$$
u(\widehat{T}, x) \leq 1+\frac{\varepsilon}{2}<\widehat{q}_{r}^{*}(\widehat{x}-x) \quad \text { for } x \in[0, h(\widehat{T})] .
$$

Define

$$
\widehat{w}(t, x):=\widehat{q}_{r}^{*}\left(\widehat{c}_{r}^{*} t+\widehat{x}-x\right) \quad \text { for } x \in\left[0, \widehat{c}_{r}^{*} t+\widehat{x}\right], t>0 .
$$

Then $\left(\widehat{w}, \widehat{c}_{r}^{*} t+\widehat{x}\right)$ is an upper solution of $P_{1}$ on $\{(t, x) \mid x \in[0, h(t+\widehat{T})], t>0\}$, and by the comparison principle (cf. 6, 7]) we have

$$
h(t+\widehat{T}) \leq \widehat{c}_{r}^{*} t+\widehat{x}, \quad u(t+\widehat{T}, x) \leq \widehat{w}(t, x) \quad \text { for } x \in[0, h(t+\widehat{T})] \text { and } t>0 .
$$

This implies that

$$
\limsup _{t \rightarrow \infty} \frac{h(t)}{t} \leq \widehat{c}_{r}^{*}
$$

Since the limits (2.10) and (2.11) hold for any small $\varepsilon>0$, we have

$$
\lim _{t \rightarrow \infty} \frac{h(t)}{t}=c_{r}^{*}
$$

by Proposition 2.5. The leftward spreading speed

$$
\lim _{t \rightarrow \infty} \frac{-g(t)}{t}=c_{l}^{*}
$$

is proved similarly.

In [6, 7], the authors considered problem $\left(P_{0}\right)$, that is, problem $\left(P_{1}\right)$ without advection term (i.e., $\beta=0$ ). Among others, they showed that the asymptotic spreading speed is characterized by the following problem:

$$
\frac{\mathrm{d} p}{\mathrm{~d} q}=\frac{c}{d}-\frac{f(q)}{d p} \quad(q<1), \quad p\left(1^{-}\right)=0 .
$$

Using a similar phase plane analysis as above, the authors in 7 proved that problem (2.12) has a solution $\left(c, P^{c}(q)\right)$ for each $c \in[0,2 \sqrt{d})$. Moreover, they proved that $P^{c}(0) \searrow 0$ as $c \nearrow c^{0}:=2 \sqrt{d}$; (2.12) has a unique solution $\left(c^{*}, P^{c^{*}}(q)\right)$ such that $\mu P^{c^{*}}(0)=c^{*}$. This $c^{*}$ is nothing but the rightward and leftward spreading speeds (cf. [6, 7]).

Combining the above phase plane analysis we have the following conclusions:

(1) $P_{l}^{c-\beta}(0)=P^{c}(0)=P_{r}^{c+\beta}(0)$ for all $c \in[\beta, 2 \sqrt{d})$;

(2) $P_{l}^{c}(0)$ (resp. $\left.P^{c}(0), P_{r}^{c}(0)\right)$ is continuous and strictly decreasing in $c \in$ $[0,2 \sqrt{d}-\beta]($ resp. $c \in[0,2 \sqrt{d}], c \in[0,2 \sqrt{d}+\beta])$.

Define three new functions $\gamma_{r}(c), \gamma(c)$ and $\gamma_{l}(c)$ by

$$
\gamma_{r}(c):=P_{r}^{c}(0) \text { for } c \in[0,2 \sqrt{d}+\beta), \quad \gamma(c):=P^{c}(0) \text { for } c \in[0,2 \sqrt{d})
$$

and

$$
\gamma_{l}(c):=P_{l}^{c}(0) \text { for } c \in[0,2 \sqrt{d}-\beta) .
$$

Then, in the $c, \gamma$-plane their graphs lie in the first quadrant (see Figure 1) and these graphs contact the straight line $\gamma=\frac{c}{\mu}$ at points $\left(c_{l}^{*}, \frac{c_{l}^{*}}{\mu}\right),\left(c^{*}, \frac{c^{*}}{\mu}\right)$ and $\left(c_{r}^{*}, \frac{c_{r}^{*}}{\mu}\right)$, respectively. Therefore, $c_{l}^{*}<c^{*}<c_{r}^{*}$. This completes the proof of Theorem 1.1. 


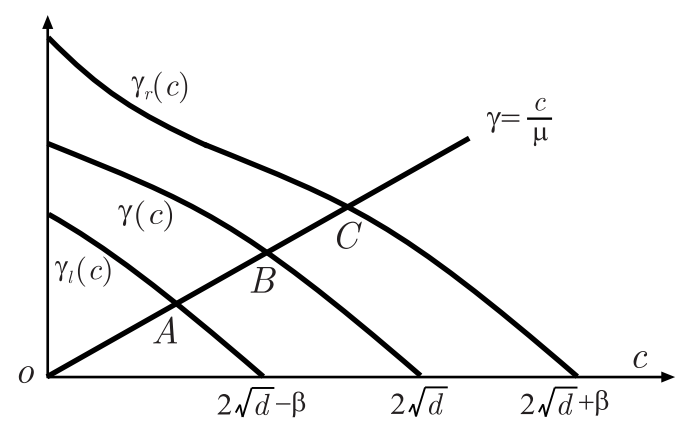

FiguRE 1. Graphs of $\gamma_{l}(c), \gamma(c), \gamma_{r}(c)$ and their contact points $A, B, C$ with the line $\gamma=\frac{c}{\mu}$; the $c$-coordinates of $A, B$ and $C$ are $c_{l}^{*}, c^{*}$ and $c_{r}^{*}$, respectively.

Proof of Theorem 1.2. The conclusions in Theorem 1.2 follow from a simple analysis on the relations among the graphs of $\gamma_{r}(c), \gamma(c), \gamma_{l}(c)$ and $c / \mu$ in Figure 1.

\section{REFERENCES}

[1] Isabel E. Averill, The effect of intermediate advection on two competing species, ProQuest LLC, Ann Arbor, MI. Thesis (Ph.D.), The Ohio State University, 2011. MR3004385

[2] D. G. Aronson and H. F. Weinberger, Nonlinear diffusion in population genetics, combustion, and nerve pulse propagation, Partial differential equations and related topics (Program, Tulane Univ., New Orleans, La., 1974), Lecture Notes in Math., Vol. 446, Springer, Berlin, 1975, pp. 5-49. MR0427837 (55 \#867)

[3] D. G. Aronson and H. F. Weinberger, Multidimensional nonlinear diffusion arising in population genetics, Adv. in Math. 30 (1978), no. 1, 33-76, DOI 10.1016/0001-8708(78)90130-5. MR.511740 (80a:35013)

[4] Yihong Du and Zongming Guo, Spreading-vanishing dichotomy in a diffusive logistic model with a free boundary, II, J. Differential Equations 250 (2011), no. 12, 4336-4366, DOI 10.1016/j.jde.2011.02.011. MR2793257 (2012c:35484)

[5] Yihong Du and Zongming Guo, The Stefan problem for the Fisher-KPP equation, J. Differential Equations 253 (2012), no. 3, 996-1035, DOI 10.1016/j.jde.2012.04.014. MR2922661

[6] Yihong Du and Zhigui Lin, Spreading-vanishing dichotomy in the diffusive logistic model with a free boundary, SIAM J. Math. Anal. 42 (2010), no. 1, 377-405, DOI 10.1137/090771089. MR2607347 (2011b:35575)

[7] Yihong Du and Bendong Lou, Spreading and vanishing in nonlinear diffusion problems with free boundaries, J. Eur. Math. Soc. (to appear) (arXiv:1301.5373)

[8] H. Gu, Zhigui Lin and Bendong Lou, Long time behavior of solutions of a diffusionadvection logistic model with free boundaries, Appl. Math. Lett. 37 (2014), 49-53, DOI 10.1016/j.aml.2014.05.015. MR.3231725

[9] Norberto Aníbal Maidana and Hyun Mo Yang, Spatial spreading of West Nile Virus described by traveling waves, J. Theoret. Biol. 258 (2009), no. 3, 403-417, DOI 10.1016/j.jtbi.2008.12.032. MR 2973250

[10] I. G. Petrovski, Ordinary differential equations, Prentice-Hall Inc., Englewood Cliffs, N.J., 1966. Revised English edition. Translated from the Russian and edited by Richard A. Silverman. MR0193298 (33 \#1518) 
[11] Aizik I. Volpert, Vitaly A. Volpert, and Vladimir A. Volpert, Traveling wave solutions of parabolic systems, Translations of Mathematical Monographs, vol. 140, American Mathematical Society, Providence, RI, 1994. Translated from the Russian manuscript by James F. Heyda. MR 1297766 (96c:35092)

Department of Mathematics, Tongui University, Shanghai 200092, People's Republic OF CHINA

E-mail address: honggu87@126.com

School of Mathematical Science, Yangzhou University, Yangzhou 225002, People's Republic of ChinA

E-mail address: zglin68@hotmail.com

Department of Mathematics, Tongui University, Shanghai 200092, People's Republic OF CHINA

E-mail address: blou@tongji.edu.cn 